\title{
Agnès Cousson, L'Écriture de soi. Lettres et récits autobiographiques des religieuses de Port-Royal
}

Angélique et Agnès Arnauld.

Angélique de Saint-Jean Arnauld d'Andilly.

Jacqueline Pascal.

Préface par Philippe Sellier, Paris, Honoré Champion, 2012, coll.

«Lumière classique », 640 p.

\section{Daniel Vidal}

\section{(2) OpenEdition \\ Journals}

\section{Édition électronique}

URL : http://journals.openedition.org/assr/25442

DOI : $10.4000 /$ assr. 25442

ISSN : $1777-5825$

Éditeur

Éditions de l'EHESS

Édition imprimée

Date de publication : 30 décembre 2013

Pagination : 165

ISSN : 0335-5985

Référence électronique

Daniel Vidal, «Agnès Cousson, L'Écriture de soi. Lettres et récits autobiographiques des religieuses de Port-Royal », Archives de sciences sociales des religions [En ligne], 164 | 2013, mis en ligne le 19 février 2014, consulté le 21 septembre 2020. URL : http://journals.openedition.org/assr/25442 ; DOI : https:// doi.org/10.4000/assr.25442

Ce document a été généré automatiquement le 21 septembre 2020

(C) Archives de sciences sociales des religions 


\section{Agnès Cousson, L'Écriture de soi. Lettres et récits autobiographiques des religieuses de Port-Royal}

Angélique et Agnès Arnauld.

Angélique de Saint-Jean Arnauld d'Andilly.

Jacqueline Pascal.

Préface par Philippe Sellier, Paris, Honoré Champion, 2012, coll.

«Lumière classique », $640 \mathrm{p}$.

Daniel Vidal

\section{RÉFÉRENCE}

Agnès Cousson, L'Écriture de soi. Lettres et récits autobiographiques des religieuses de Port-Royal

Angélique et Agnès Arnauld.

Angélique de Saint-Jean Arnauld d'Andilly.

Jacqueline Pascal.

Préface par Philippe Sellier, Paris, Honoré Champion, 2012, coll. « Lumière classique », $640 \mathrm{p}$. 
1 À la sœur Marie de Saint-Joseph, la mère Agnès, abbesse de Port-Royal, rappelle "qu'il faut entrer dans les choses de Dieu si purement, qu'on n'y contribue rien que le simple consentement et acquiescement à ce qu'il veut faire en nous ». La Règle du couvent, en effet, héritière exigeante de la culture augustinienne, tout entière vouée à la seule œuvre de Dieu, porte discrédit du «moi», cette protestation d'amourpropre qui fait obstacle à son accomplissement. Et discrédit doit s'entendre en son sens le plus radical, qu'Agnès Cousson décline en toutes ses occurrences. Dans l'espace de Port-Royal, et au sein de la communauté des religieuses, tout doit s'effacer qui pourrait relever d'une singularité affective, d'une présence personnalisée, d'une identité spécifique. Le saccage du «moi» est

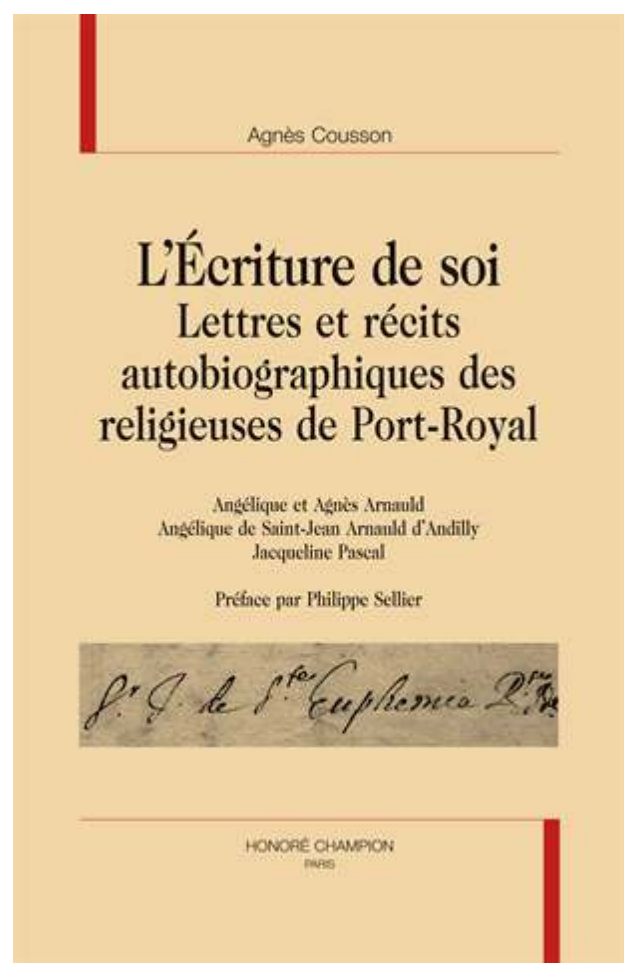
poursuivi jusqu'à son anéantissement même. Les moniales sont esclaves de Dieu, victimes sacrificielles d'un holocauste spirituel. Clôture conventuelle et clôture spirituelle définissent alors un haut lieu d'expérience entièrement dévolu à Dieu, où les religieuses ne sont tant «mortes au monde », qu'en elles-mêmes le monde se meurt. Monde du silence, car la parole est porteuse de corruption et source de péché. Sait-on que dès le début de l'ordre de Citeaux, les religieux communiquaient en langue des signes, muets comme ces « incirconcis des lèvres » dont parle l'Écriture, et sourds à ce qui pourrait s'entendre de leur monde intérieur? Lavés ainsi de tout soupçon d'effusion et de plaisir propre à l'échange. À Port-Royal, le silence est la loi. Si l'on «parle ", cependant, ce n'est que sous condition de spiritualité ou de morale, en un acte où rien, en somme, ne s'échange qui ne soit contrôlé par un rappel constant de la Règle. L'auteur examine avec précision l'ensemble des interdits qui organisent la «soumission muette » des religieuses, et qui décident de leur réclusion en une solitude intérieure où l'on peut alors mourir à soimême. Demeurer en l'esprit d'oraison, suppose que l'on se garde de toute « dimension affective ", que l'on habite un "vide intérieur » qui fasse accueil à Dieu seul. Ablation de soi pour oblation à Dieu. En toutes choses, ne ressentir plaisir ni peine, mortifier son corps sans que souffrir soit occasion de dolorisme. Servitude volontaire: n'avoir volonté que de vouloir ne pas faire ce que l'on veut - partout, s'opposer à soi-même. Faire humilité, ne compter pour rien. Précis de désappropriation, programme de décomposition de soi.

2 Dans ce lieu de spiritualité voué à la gloire de Dieu et à sa seule obéissance et sa seule volonté, si l'on ne communique par la parole qu'au prix de soi et à son propre péril, puisque la corruption est logée au cœur du langage comme son ombre tentatrice, on écrit, par contre, à foison. Dans les seules bibliothèques parisiennes, Agnès Cousson a pu exploiter près de trois mille Lettres, qui s'ajoutent aux divers Récits de captivité rédigés en 1665 par les religieuses ayant refusé de signer le Formulaire, et des Vies 
(dont celles d'Angélique de Saint-Jean), et des Relations (dont celle d'Angélique Arnaud). Le recours à la lettre permet-il de se libérer des contraintes auxquelles est assujettie la communication orale? L'écrit, cette «communication Ad Extra » Permet-Il De Briser Avec La Clôture Spirituelle ? À Port-Royal, On N'écrit Pas Sans Obéir Scrupuleusement A L'impératif De S'abstraire De Toute Subjectivité Et Affectivité. G. Gusdorf, Commentant Pascal, Rappelait Qu'« Une Vie Humaine Ne Possède Pas Son Centre En Elle-Même, Ni Sa Valeur Intrinsèque ». L'écriture Ne Saurait Alors Relever De Quelque Expression De Soi, Et De Quelque Réappropriation De Ce Qui Fut Mis Radicalement En Perte. Après Avoir Précisé, A Partir D'une Analyse Approfondie Des Lettres Des Religieuses, Les Conditions Strictes De La Communication, L'auteur Examine Comment L'observance Même De Ces Contraintes Se Heurte A Des Ambiguïtés Fondamentales. L'écriture De Port-Royal Est Ecriture De Soumission A La Règle, En Même Temps Que De Son Contournement. C'est Sans Doute En La Mise En Evidence De Ce Paradoxe Et De Sa Richesse D'enseignement Que Réside Le Meilleur De L'ouvrage. Ainsi De La Manière Dont Les Religieuses Considèrent Le Cœur. Il Est La Demeure De Dieu Et « Le Point De L'être Où Il Convient De Se Retrancher » Pour Le Chercher. Mais Si Le Cœur Est Bien Cela, Cette Intériorité Qui Qualifie, Comme On Dira Plus Tard, "Le Chrétien Intérieur », Alors Approcher Le Fond Du Cœur Est Approcher Cet Espace D'intimité Dans Lequel Il Faut « Descendre Et S'enfoncer ». Intimité ? Le Concept Même Est «Effrayant », Et C'est Cependant A Cet Effroi Que Le Sujet Est Confronté. L'écriture Borde Cet Effroi, Si Elle Ne Le Dit Pas. Elle $\mathrm{Ne}$ Se Fait Pas Introspection Globale, Et $\mathrm{Ne}$ Tolère Qu'une «Connaissance Approximative » De Soi. Il N'empêche : La Règle Ne Peut Valoir Que Si L'écriture La Met Un Instant En Suspens. Ou Si Elle La Prend Au Mot, Et En Subvertit La Rigueur. Il Faut, Dit Egalement La Règle, S'oublier. Mais S'oublier Suppose Que L'on Sache Ce Que L'on Est, Et De Quelle Faute On Est Coupable, Pour S'affranchir De Ce «Vieil Homme » Que L'on Doit Mettre A Mort. Il Faut Donc «S'examiner Pour Connaître L'origine De La Faute ». Atteindre Le «Vide Intérieur » Implique Que L'on Demeure, Fût-Ce De Façon Fugitive, Dans Cet Univers Morbide De La Faute, Qui Est, Pour La "Créature », La Condition Même De Son Existence.

3 Les écrits de Port-Royal disent en une même phrase la loi et sa transgression. « Parce qu'il doit s'effacer, le "moi" ne peut s'oublier ", écrit l'auteur. Ne doit s'oublier, peut-on dire. Étrange disposition de l'écriture, qui pose à la fois l'impératif de la perte et la nécessité de la présence. Qui instaure un rapport à soi gouverné par l'économie du soupçon. En effet, l'extrême dépossession de son «moi » requiert sa pleine prise en charge. Prendre en charge sa «faute » participe de cette plongée en l'intimité que la Règle interdit. Mais plonger en son intériorité est entrer la demeure de Dieu dit la Règle. Écrire « selon son humeur » est une faute, dit encore la Règle, qui récuse toute expression de sentiments. Mais en cette nécessité de se savoir coupable, il ne s'agit pas de sentiments comme il en irait d'une effusion lyrique. Il s'agit de ce qui institue en profondeur ce que l'auteur appelle le sujet. « Le contrôle de soi ramène le sujet à luimême ». Ce que la Règle exige, elle en condamne l'effectivité. Dieu n'est qu'au prix de l'affirmation du sujet. Les lettres de Port-Royal ne cessent d'effacer le sujet de l'écriture ? C'est pour mieux, semble-t-il, l'affirmer. Aussi bien la lettre, à Port-Royal, introduit un " espace de relative liberté » où peuvent se livrer quelques «sentiments humains", et se nouer quelque amitié affective dans l'affirmation d'une pure spiritualité. La lettre, ou la possibilité improbable d'un « dialogue intérieur ». Et d'un "récit personnel » et son "effet individualisant». Non de façon directe, qui ferait objection insoutenable à la Règle. Mais par des chemins obliques. Si elle parle d'elle 
" pour des raisons autres que spirituelles ", telle religieuse préférera l'indéfini « on ", « nous » au " je ». Toute une stratégie est mobilisée de dépersonnalisation de l'énoncé, d'évitement du «je », qui permet à la fois de demeurer dans le cadre de la Règle, et d'affirmer qu'un autre énonciateur est possible. Tant tourne-t-on en effet autour du sujet que le sujet devient alors la question en suspens (en abîme?) au cœur de l'écriture, par quoi elle se fonde et se déploie. 